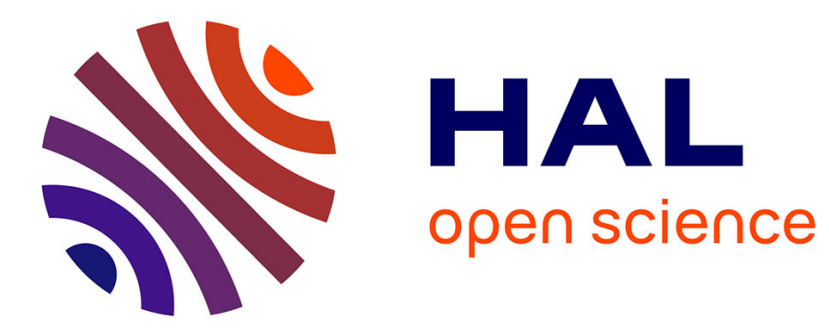

\title{
Langue et apprentissage de la lecture au Japon \\ Christian Galan
}

\section{To cite this version:}

Christian Galan. Langue et apprentissage de la lecture au Japon. L'information grammaticale, 2012, 133 (1), pp.41-50. 10.3406/igram.2012.4201 . hal-03203687

\section{HAL Id: hal-03203687 \\ https://hal.science/hal-03203687}

Submitted on 21 Apr 2021

HAL is a multi-disciplinary open access archive for the deposit and dissemination of scientific research documents, whether they are published or not. The documents may come from teaching and research institutions in France or abroad, or from public or private research centers.
L'archive ouverte pluridisciplinaire HAL, est destinée au dépôt et à la diffusion de documents scientifiques de niveau recherche, publiés ou non, émanant des établissements d'enseignement et de recherche français ou étrangers, des laboratoires publics ou privés. 


\section{LANGUE ET APPRENTISSAGE DE LA LECTURE AU JAPON}

\section{Christian GALAN}

Un des défis majeurs de l'école japonaise est de faire acquérir à l'ensemble de ses élèves la maîtrise de la lecture dans une langue écrite considérée comme l'une des plus complexes du monde 1 . Cette école y parvient sans difficulté majeure puisque, même s'il est difficile d'accepter l'idée que ce pays ne compterait pas d'«illettrés» (Galan, 2006), force est de reconnaître que le Japon se classe plutôt bien dans les enquêtes internationales sur la maîtrise scolaire de la lecture, et que personne ne considère dans ce pays que l'accès à la lecture puisse constituer un quelconque problème pour tout enfant normalement constitué. Et si débats il y a, quant à la nature ou à la qualité de l'enseignement de la lecture, ils ne ressemblent en rien aux violentes querelles que l'on connaît dans nos pays occidentaux.

Après un rapide rappel de la nature du système d'écriture japonais, nous présenterons la méthode d'enseignement de la lecture appliquée aujourd'hui dans les écoles de l'archipel, puis nous aborderons trois aspects de cet enseignement que l'exemple japonais nous semble particulièrement bien mettre en valeur, à savoir : ce qui dans la méthode d'enseignement ou d'apprentissage retenue est «imposé » (ou pas) par la langue; ce qui fait que la «méthode» est devenue ce qu'elle est, c'est-à-dire la manière dont elle s'est mise en place (déterminants et choix historiques, pédagogiques, linguistiques, etc.); enfin, en lien avec les deux points précédents, nous interrogerons le concept même de «méthode » en examinant le rapport qui existe entre ce que l'on désigne comme tel et ce qui relève de la pratique des maîtres.

Nous conclurons en nous intéressant aux derniers développements qu'a connus le débat sur l'enseignement de la lecture au Japon depuis les années 2000 en présentant les changements les plus récents qui ont affecté cet enseignement.

\section{LE SYSTÈME D'ÉCRITURE DU JAPONAIS}

Nous ne pouvons pas présenter ici en détail la nature et le fonctionnement du système d'écriture du japonais (Galan, 2003; Tamba, 2006), pas plus que l'histoire de son évolution

1. Cet article synthétise l'ensemble des travaux que nous avons menés sur l'enseignement actuel de la lecture au Japon, notamment dans les sections 1 et 2 (Galan, 1995a, 1995b; Galan et Fijalkow, 2006). La section 3 et la conclusion développent de nouveaux éléments de réflexion sur cet enseignement tout en en présentant les évolutions les plus récentes. et de la manière dont il est parvenu à son état actuel, et nous renverrons pour cela aux travaux existants (Galan, 2001a; Frellesvig, 2010; Blin et Tamba, 2001). II nous semble toutefois indispensable de commencer par rappeler quelques-unes des caractéristiques les plus importantes du système d'écriture du japonais.

Nous rappellerons que la syllabe, bien qu'analysable en phonèmes comme dans toutes les langues, occupe en japonais une place si fondamentale qu'elle peut être considérée sur le plan phonologique, et par voie de conséquence sur le plan graphique, comme l'unité principale de cette langue.

Le système d'écriture de la langue japonaise combine aujourd'hui deux systèmes de transcription fondamentalement différents : les kana (hiragana et katakana), qui sont deux systèmes syllabiques, phonétiques et combinatoires, de 46 signes chacun à la correspondance phonie-graphie quasi parfaite ( 1 signe = 1 son), et les kanji, de nature sémiographique, dont le nombre s'élève à plusieurs milliers, et qui sont en fait les caractères chinois dans leur usage japonais, c'est-à-dire des éléments graphiques complexes porteurs chacun de plusieurs sens et de plusieurs sons.

$$
\begin{gathered}
\text { トうきょうキョウ } \\
\text { Figure 1. } \\
\text { Le même mot, Tôkyô, transcrit de gauche à droite } \\
\text { en hiragana, en katakana et en kanji }
\end{gathered}
$$

Cette mixité est la grande caractéristique de l'écriture japonaise, ce qui la distingue fondamentalement de tous les autres systèmes d'écriture. Certains mots sont ainsi écrits entièrement en kanji (mots conceptuels et patronymes; par exemple : 文法 bunpô, «grammaire »), d'autres entièrement en hiragana (mots de coordination, verbes auxiliaires, démonstratifs, particules grammaticales, etc.; par exemple : それから sorekara, “ et ensuite»), d'autres, enfin, grâce à une combinaison des deux (par exemple : 高い takai, « haut»). Dans ce dernier cas, qui concerne essentiellement les mots variables (verbes et adjectifs), la racine du mot porteuse de sens est en général écrite en kanji (高 : «haut») et les hiragana placés à sa suite servent à noter les différents suffixes (い : neutre, non accompli, affirmatif). Quant aux katakana, leur utilisation dans le système d'écriture actuel se limite à des cas bien précis, dont le principal est la transcription des mots étrangers non chinois (par exemple, パリ : Paris; ジョン: John). 
Les hiragana et les katakana, qui rendent compte des mêmes sons, ne signifient rien en eux-mêmes mais peuvent produire du sens en fonction de leur nombre et de l'ordre dans lequel ils se présentent. Ils couvrent la totalité des possibilités phonologiques de la langue japonaise et permettent, à eux seuls, de transcrire celle-ci, sans avoir recours aux kanji. Mais ce n'est pas là, pour autant, l'usage «normal » ou « adulte », car un texte transcrit uniquement en kana devient difficile à segmenter et à oraliser; il perd en efficacité et sa lecture devient plus incertaine. II n'existe pas en effet en japonais de blanc graphique pour séparer les mots, et le lecteur, dont l'œil n'accroche plus sur les «taches» plus sombres des mots écrits en kanji, voit sa lecture considérablement ralentie (sans parler des très nombreux homophones que la transcription à l'aide des seuls hiragana ne permet plus de distinguer).

$$
\begin{gathered}
\text { 私は三月に日本へ行きます。 } \\
\text { わたしはさんがつにほんへいきます。 }
\end{gathered}
$$

Figure 2. La même phrase (Watashi wa sangatsu ni nihon e ikimasuJ'irai au Japon au mois de mars) transcrite tout d'abord «normalement », puis à l'aide des seuls hiragana.

Chaque kana étant associé à un son et à un seul, les règles liant écrit et oral sont, en ce qui concerne les deux syllabaires, extrêmement fiables. Ainsi, une fois qu'il connaît les 46 hiragana (plus quelques règles très simples concernant la transcription des sons dits «complexes » : allongements, diphtongues, sons voisés, etc.), un enfant japonais peut écrire tous les mots qu'il entend, même s'il ne les a jamais rencontrés écrits auparavant: il lui suffit de retrouver les signes correspondant aux syllabes entendues et il n'y en a qu'un seul pour chacune d'entre elles.

Concernant les kanji, rappelons qu'à la différence des kana ils représentent un ou plusieurs sens et possèdent, le plus souvent, plusieurs lectures qui varient en fonction du contexte de sens dans lequel ils apparaissent et de leur environnement graphique : kanji et/ou kana précédents et/ ou suivants.

Comme dans le cas des caractères chinois, on les classe en quatre grandes catégories : les pictogrammes (日: soleil; 月: lune), les idéogrammes (一 : un; 上 : dessus), les caractères composites (combinaisons d'éléments des deux catégories précédentes; 明 $(\boxminus+$ 月) = lumière, clarté) et les caractères phonétiques, c'est-à-dire des caractères formés d'un élément phonétique et d'un élément donnant une indication souvent très vague (homme, métal, animal, etc.) du champ sémantique auquel il appartient (梅: 木 arbre + 毎 bai = l'arbre dont le nom est bai, le prunier). Ce sont ces derniers caractères qui sont, et de loin, les plus nombreux puisqu'ils représentent environ $90 \%$ de l'ensemble des caractères chinois et des kanji, rendant complètement impropre l'utilisation du terme «idéogramme » pour désigner l'ensemble de ces signes.

Dans le même ordre d'idée, le point de vue selon lequel les caractères chinois, et donc les kanji, permettraient un accès direct au sens, via la seule graphie, n'est plus de mise : on sait aujourd'hui que, les concernant, la phonologie joue aussi un rôle important dans l'accès au sens, y compris dans la lecture silencieuse ou le langage interne - même si on se demande encore lequel : «route» obligatoire, activation automatique ou «simple» rôle d'«aide»? (Galan, 2001b, 2001c, 2008).

Sur le plan graphique enfin, chaque kanji est composé d'éléments distincts plus ou moins complexes qui, agencés différemment, entrent aussi dans la composition d'autres caractères. Leur tracé s'effectue selon un ensemble de règles strictes et précises, du respect desquelles dépendent non seulement l'équilibre sinon la beauté graphiques des caractères, mais aussi, et surtout, vu le nombre élevé des traits entrant dans la composition de certains d'entre eux, leur lisibilité.

Même si le nombre des kanji «d'usage courant» et celui de leurs lectures ont été respectivement limités, après la fin de la Seconde Guerre mondiale, à 1945 et à 4 084, un Japonais se doit toutefois de connaître au minimum 3000 à 4000 caractères pour pouvoir prétendre accéder librement à l'écrit...

Enfin, dernière caractéristique du japonais écrit qu'il convient de rappeler ici, mais pas la moins importante, même si elle est souvent oubliée dans les descriptions de cette langue : tout scripteur a, à tout moment, la liberté de transcrire en hiragana (pour des raisons d'économie, de rapidité, de style, de difficulté ou encore d'oubli) tel ou tel mot qui, «normalement (mais il s'agit d'une norme non écrite et que personne ne peut expliquer), devrait se transcrire dans l'écriture «adulte» en kanji. Et cela, sans que le sens de l'énoncé en soit affecté, sans que cela provoque la moindre gêne pour le lecteur. II est donc difficile de dire, comme on le dit souvent, que les kanji notent le «sens», et les kana, «la langue », ces derniers participant eux aussi à la notation du sens (Galan 2008).

\section{LA STRATÉGIE D’ENSEIGNEMENT DU JAPONAIS ÉCRIT}

Trois mots peuvent résumer la stratégie d'enseignement de l'écrit que les Japonais ont mise en place dans leurs écoles : «mémorisation», «accumulation » et «substitution» : mémorisation préalable des hiragana (et des katakana), mémorisation et accumulation de plusieurs centaines de kanji (lecture(s) et écriture) étalées dans le temps (1006 durant les 6 années de l'école élémentaire), et substitution progressive des kanji étudiés aux hiragana dans les phrases écrites ou rencontrées par les apprenants2. Ajoutons au sujet de ce dernier point que, s'il se trouve quelques caractères qui apparaissent dans un texte alors qu'ils n'ont pas été étudiés, ils sont alors accompagnés de furigana, c'est-à-dire de hiragana tracés, en petit, au-dessus ou sur le côté des kanji, afin de préciser la lecture de ceux-ci. Peu fréquents

2. Pour une description résumée de cette stratégie d'enseignement voir Galan (1995). Pour une description détaillée, ainsi que pour l'histoire de la mise en place de stratégie, voir Galan (2001a) et Shutô (2006). 
dans les manuels scolaires, ils ne sont que rarement utilisés dans un but pédagogique.

Les directives d'enseignement du ministère de l'Éducation (gakushû shidô yôryô) imposent une programmation systématique et une progression synthétique de l'apprentissage de la lecture. Tous les points inclus dans les programmes doivent être traités sans exception et chacun d'entre eux prédéfinit, pour un domaine ou un savoir-faire donnés, un palier à franchir dans la maîtrise de ce savoir-faire ou de l'une des compétences qui se rattache au domaine en question.

Ces directives ne parlent nulle part de manuels scolaires (kyôkasho) et font simplement référence au terme plus vague de kyôzai, matériel pédagogique. Les instituteurs sont toutefois tenus - au risque de se mettre, dans le plein sens du terme, «hors la loi »-d'utiliser un manuel pour chacune des matières qu'ils enseignent, et donc, bien sûr, pour la matière «langue japonaise» (kokugo), au sein de laquelle est mis en place l'enseignement de la lecture. Aucun auteur, aucune maison d'édition ne peut publier librement son propre manuel; seuls peuvent être publiés les manuels autorisés par le ministère de l'Éducation. Ils sont distribués gratuitement aux enfants pendant toute la durée de la scolarité obligatoire, mais leur choix, déjà limité aux séries des seules maisons d'édition autorisées, ne relève ni de l'instituteur, ni de l'école, mais d'une décision de l'autorité éducative locale qui vaut pour l'ensemble de la zone éducative considérée 3.

Si les directives du ministère ne traitent ni de la pédagogie à appliquer, ni des apprentissages à mettre en place pour parvenir à la réalisation des objectifs qu'elles visent, les instituteurs ne sont pas pour autant libres de conduire leur classe comme ils l'entendent. L'obligation qui leur est faite d'utiliser les seuls manuels autorisés revient en effet à leur imposer à tous une «méthode» unique. Ainsi, au nombre d'heures de cours de langue prévu par les programmes, correspond dans les manuels, pour chaque année, un nombre équivalent de leçons dont le contenu est fixé de façon à ce que, à la fin de l'année, tous les points apparaissant dans les directives aient bien été étudiés et toutes les pages du manuels... utilisées. De fait, «les manuels font méthode », même si peu de gens au Japon le diraient ainsi pour ce qui est de l'enseignement de la lecture.

À la différence des pays occidentaux, où les tenants des différentes méthodes de lecture acceptent en général, malgré leurs désaccords, l'idée que si toutes les méthodes peuvent fonctionner aucune cependant ne fonctionne pour tous les enfants en même temps, et que c'est en dernier ressort à l'enseignant de trancher en fonction des réalités de sa classe, au Japon, en revanche, ce point de vue n'a pas cours. C'est même un souci totalement opposé qui anime les responsables de l'éducation de ce pays, et qui fonde la pédagogie des maîtres, puisque leur volonté affichée est de parvenir à ce que tous les enfants d'une même classe, et toutes les classes d'un même niveau, d'une même école, d'une même zone géographique et, dans l'absolu, du

3. Sur les manuels de lecture, voir Tsukada (2006) pays tout entier, progressent d'un même pas et reçoivent un enseignement le plus identique possible. La latitude laissée aux enseignants, sinon de choisir leur pédagogie, du moins d'adapter individuellement celle qui leur est imposée à la réalité de leur classe y est des plus réduite 4 . De fait, l'immense majorité des instituteurs japonais suit scrupuleusement la progression et le contenu des directives du ministère et des manuels. C'est, pourtant, au niveau de cette (faible) marge de manœuvre, que les Japonais parlent le plus facilement de «méthode» (hôhô). On y reviendra.

Arrêtons-nous encore un instant sur la progression retenue pour l'enseignement de la lecture et de l'écriture.

Le premier trimestre de la première année est consacré aux hiragana, que la quasi-totalité des enfants connaissent pourtant déjà avant leur entrée à l'école élémentaire, et qui sont (re) présentés un par un (ou deux par deux) au cours de leçons se déroulant toutes sur un même modèle : une leçon = un son + le signe correspondant et son tracé + combinaison avec les signes déjà étudiés + exercices d'imitation, de répétition, et récapitulations fréquentes. À la veille des vacances d'été, soit après trois mois de ce régime (la rentrée a lieu au Japon en avril), la grande majorité des enfants des classes de 1re année (âgés donc de 7 ans) sont capables de s'exprimer par écrit à l'aide de phrases ou de petits textes rédigés dans un japonais «correct» et compréhensible par tous, ainsi que de lire/déchiffrer n'importe quel texte écrit en hiragana.

Les deux trimestres suivants sont consacrés à l'apprentissage du second syllabaire katakana et des premiers kanji, ainsi qu'aux activités de lecture dont les textes servent de support à l'étude de ces derniers. Les séquences sont bâties plus ou moins sur le même modèle avec pour support un des récits figurant dans le manuel : préparation du texte à la maison (beaucoup d'enfants le connaissent d'ailleurs par cœur), début et fin du cours par la lecture collective à haute voix de la partie du texte étudiée - voire du texte dans son entier -, explication des mots «difficiles» par des synonymes ou des périphrases, questions-réponses sur le sens du texte entre l'enseignant et les élèves, etc.; ce qui est écrit au tableau durant les leçons de lecture (et plus généralement de langue) est toujours présenté de la même façon, qui est celle que l'on retrouve, identique, dans toutes les classes de la première à la sixième année (l'école élémentaire japonaise compte six années) et qui est aussi celle des tests, des carnets d'exercices, ainsi que celle des cahiers des enfants, qui prennent très rapidement l'habitude, dès cette première année, de recopier tel quel ce que le maître écrit au tableau.

4. Certes, comme on le verra dans notre conclusion, le ministère de l'Éducation japonais prône officiellement, depuis quelques années, un enseignement plus individualisé et plus différencié. Toutefois, pour le sujet qui nous intéresse ici, on a encore du mal à voir, à défaut de ce qui aurait changé, ce qui pourrait changer, étant donné, entre autres, que les directives ont toujours valeur de loi, que l'usage des manuels scolaires (et la « méthode» dont ils sont porteurs) restent obligatoires, ou encore que la progression au sein des classes reste collective. Bref, que le cadre général, structurel, ainsi que la pratique professionnelle contredisent le discours officiel. A l'évidence, si une évolution est possible, elle est encore à venir. 
La première année voit ainsi se mettre en place ce que l'on pourrait appeler une «routine » de travail concernant l'étude des signes et les leçons de lecture. L'objectif est de faire acquérir aux enfants un certain nombre d'habitudes et de réflexes dans leur façon d'étudier, en classe ou à la maison (notamment pour ce qui est des kana puis des kanji), et de fixer, sinon de figer, les leçons de lecture dans un cadre général (structure, déroulement des leçons et habitudes de travail) qui ne variera guère au cours des cinq années suivantes.

De fait, en ce qui concerne l'enseignement des kanji, on distingue clairement trois temps durant les six années de l'école élémentaire. Dans un premier temps, durant la $1^{\text {re }}$ année, il s'agit de bien faire comprendre aux enfants comment ces kanji fonctionnent et comment on doit les étudier. Les instituteurs passent en général beaucoup de temps sur chaque signe et seulement 80 sont étudiés durant cette année-là (contre 160 à 220 les années suivantes). Ils le sont sous tous leurs aspects possibles, l'accent étant mis sur leur composante graphique (ordre du tracé des traits) et phonique, avec une insistance particulière sur leur iconicité et le rappel de leur «étymologie - à comprendre ici comme leurs formes anciennes et surtout originelles (voir : Kawakami, 2006). Quand les caractères sont composés de plusieurs éléments graphiques, ils sont décomposés et chaque élément est étudié (sens, autres kanji dans lesquels on le retrouve, etc.). Cet exercice est d'autant plus «simple » à mettre en place que les kanji étudiés sont majoritairement des pictogrammes et des idéogrammes (plus de la moitié des $80 \mathrm{kanji}$, alors que ceux-ci, on l'a vu, représentent moins de $10 \%$ de l'ensemble des kanji), et que beaucoup sont aussi des caractères simples entrant dans la composition d'autres caractères graphiquement plus complexes. La routine d'apprentissage se met également en place concernant le travail personnel demandé à la maison : "colonnes » d'écriture (de signes ou de mots composés de plusieurs signes); exercices consistant tantôt à noter, dans la marge de phrases où apparaissent des kanji, la lecture de ceux-ci en hiragana, tantôt à écrire, dans des espaces laissés vides dans des phrases, les kanji correspondant aux lectures écrites en hiragana dans la marge.

Dans un deuxième temps, à partir de la deuxième année, ce travail se poursuit à l'identique avec toutefois une référence de moins en moins appuyée à l'«étymologie " - ce qui se comprend, puisque la proportion de kanji «phonétiques» augmente alors que celle des pictogrammes et idéogrammes diminue considérablement, et qu'il est donc moins aisé de pratiquer cet exercice. En revanche, les kanji sont toujours étudiés par rapport à leurs éléments graphiques constitutifs, leurs clefs (éléments sémantiques), etc. La liste des kanji à étudier est fixée par les programmes et, les enseignants ne pouvant s'en écarter, les regroupements par «famille» de kanji se font donc par rapport aux kanji déjà étudiés ou, au mieux, étudiés durant l'année. La difficulté majeure de la lecture en japonais résidant dans l'oralisation des kanji, l'accent est mis en priorité sur cette activité ainsi que sur l'aspect graphique des caractères : tracé, ordre des traits. Les exercices décrits dans le paragraphe précédent continuent d'être pratiqués «en batterie » afin de faire mémoriser du mieux possible les différentes lectures des caractères, en les présentant dans un maximum de contextes sémantiques différents (certes souvent très pauvres : des phrases isolées le plus souvent), jusqu'à ce que l'oralisation ou le tracé deviennent «automatiques».

Le troisième temps, enfin, débute en général à partir de la quatrième année qui voit, à la fois, une accélération des pratiques d'apprentissage précédemment décrites et une externalisation de celles-ci : jusque-là étudiés pendant les heures de langue, les kanji relèvent à présent presque entièrement du travail personnel des enfants. L'instituteur établit en général, pour chaque semaine, une liste d'une dizaine de kanji que les élèves doivent étudier chez eux. Le travail demandé est exactement le même que celui qui était jusque-là accompli en classe dans le cadre des leçons de langue, dictionnaires et cahiers d'exercices spéciaux remplaçant l'enseignant : pour chaque nouveau kanji, les enfants doivent ainsi vérifier son origine et l'ordre du tracé de ses traits, chercher ses différentes lectures ainsi que des exemples de mots composés dans lesquels il apparaît et, pour finir, écrire plusieurs phrases à l'aide de certains de ces mots. Sans oublier, toujours, des «colonnes» et des «colonnes» d'écriture.

Même si une heure par semaine reste encore consacrée aux kanji (ceux-ci y sont étudiés sous leurs aspects les plus généraux : "familles» de kanji, clés, valeur sémantique des éléments qui les composent, etc..), l'école n'apparaît plus toutefois, à partir de ce moment-là, que comme un lieu de "vérification" de la bonne marche du processus d'apprentissage. Les contrôles se font le plus souvent à l'aide de petits tests quasi quotidiens, très courts et très rapides, glissés entre deux autres activités plus importantes, et de même nature que ceux que les enfants utilisent pour mémoriser les kanji. Des tests du même type mais plus conséquents ont lieu une ou deux fois par mois, et les enfants s'autocontrôlent, régulièrement, à l'aide d'exercices, tels que décrits précédemment, achetés dans le commerce et faits "en batterie », des dizaines et des dizaines de fois.

La «mécanique» d'apprentissage des kanji, mise en place en première année et rodée pendant les deux années suivantes sous le contrôle de l'enseignant jusqu'à devenir un réflexe ou une routine à l'apparition de chaque nouveau caractère, fonctionne maintenant toute seule et permet aux enseignants des grandes classes de se décharger de cet enseignement sur le travail personnel des enfants, avec la certitude que celui-ci sera correctement accompli, le rôle des parents, et surtout des différentes formes de "soutien extrascolaire » (juku), étant extrêmement important à ce niveau du processus d'apprentissage.

\section{QUESTIONS DE «MÉTHODE»}

\subsection{Ce qu'impose la langue...}

La «méthode» (on discutera ce terme plus loin) que nous venons de décrire est considérée aujourd'hui au Japon comme si «évidente » qu'il ne viendrait à quasiment personne 
l'idée de la remettre en cause voire simplement de la questionner. Elle repose pourtant sur des choix pédagogiques qui ont été faits à une époque donnée, et qui auraient pu être autres. C'est en tout cas ce que nous apprend l'histoire de la mise en place de cet enseignement. Mais, commençons par voir ce qu'impose véritablement la langue en tant que telle.

Tout d'abord, une évidence : contrairement à ce que l'on a longtemps dit, le sens des caractères chinois ayant été associé à ces derniers de façon arbitraire, la mise en place d'un apprentissage est nécessaire pour ceux-ci et donc également pour les kanji : le sens dont tous ces signes sont porteurs n'a a priori rien d'évident et il ne se suffit pas de les regarder pour les comprendre.

Ensuite, la mémorisation des quelques centaines d'éléments graphiques qui peuvent composer les kanji et, surtout, parmi le nombre pratiquement illimité de leurs combinaisons possibles, de celles qui constituent réellement des kanji (plusieurs milliers, dont pas un des traits qui les composent ne peut être retiré ou ajouté), implique sans objection possible - à la différence de celle des kana dont la durée d'acquisition est de l'ordre de quelques semaines - un étalement de cet apprentissage dans le temps ainsi que, de la part de l'apprenant, des efforts importants et une motivation certaine.

La lecture (l'oralisation) d'un kanji dépendant du contexte dans lequel il apparaît, une grande partie de l'apprentissage de la lecture devrait donc consister par ailleurs, à l'évidence, à faire rencontrer aux apprenants un même kanji dans le plus grand nombre d'environnements différents possibles. Lire/oraliser les kanji ne relève en effet pas d'une simple question de mémoire : un décodage phonétique correct de ceux-ci implique une bonne compréhension du sens contenu dans la séquence dans laquelle ils apparaissent. Les différentes lectures sont en effet le plus souvent porteuses de différences ou de nuances sémantiques.

Enfin, chaque caractère possédant son ordre des traits, lequel doit être mémorisé en même temps que le caractère lui-même, la «mémoire de la main » se pose en précieux auxiliaire de l'esprit, et aide ce dernier à enregistrer et à conserver des graphies d'une aussi grande complexité. L'apprentissage de la lecture est ainsi étroitement lié à celui de l'écriture.

Ajoutons encore, pour conclure sur ce point, que la nature du système graphique japonais impose également, de fait, une conception du lire et de l'apprendre à lire différente de celle qui a cours dans nos pays.

En effet, du fait de la nature des kanji et de leur nombre, l'apprentissage de la lecture devrait apparaître au Japon comme jamais terminé, n'importe quel lecteur pouvant, à tout moment, tomber sur un signe qu'il n'a jamais rencontré. Pourtant, il n'en est rien et, pour les Japonais, un enfant «sait lire» dès lors qu'il maîtrise parfaitement les hiragana et qu'il a commencé à apprendre les premiers kanji. Une différence très nette est ainsi faite entre «savoir lire » et «connaître les kanji». Si l'étude de ces derniers s'étale sur toute une vie, «savoir lire » en revanche apparaît aux parents et aux enseignants japonais comme quelque chose qui peut - et doit - être rapidement et définitivement achevé. En exagérant à peine, on pourrait dire ainsi que le «savoir lire » est atteint au Japon avant même que l'étude des kanji ait commencé.

Si l'on résume, outre ce dernier point, la langue écrite japonaise nous semble ainsi imposer à l'apprenant trois choses, et trois choses seulement : tout d'abord, du fait du nombre de signes à maîtriser, un étalement de l'apprentissage de ces signes sur un temps relativement long; ensuite, du fait de la nature particulière des kanji, le recours à des exercices répétitifs de copie indispensables à leur mémorisation; et, enfin, la nécessité de réaliser cet apprentissage au travers d'énoncés riches de sens.

Pourtant, on l'aura compris en lisant la section 2 de cet article, si les deux premières choses que nous semble imposer la langue japonaise sont bien effectivement au cœur de la méthode mise en place, la troisième, en revanche, est loin d'être toujours prise en compte. L'apprentissage mis en place dans le cadre de l'école est ainsi très technique, routinier sinon répétitif, fastidieux diront certains, et au final peu contextualisé (caractères ou mots le plus souvent étudiés à vide ou dans des phrases indépendantes les unes des autres), alors que, sur ce dernier point au moins, la langue semblerait plutôt imposer un très haut degré de contextualisation, voire interdire tout apprentissage «à vide ». L'accent est en fait mis essentiellement sur les activités de codage et de décodage. L'accès au sens se fait presque exclusivement par déchiffrage de l'écrit, et la possibilité de construire du sens directement à partir de la perception visuelle ou contextuelle d'un texte n'est que rarement envisagée (même en ce qui concerne les kanji, ce qui est pour le moins surprenant). Dans le cadre scolaire, on lit le plus souvent pour oraliser, et rarement pour agir, ressentir ou penser. De même la compréhension et l'interprétation des textes lus sont bien plus souvent collectives qu'individuelles (Galan, 2001a: 35-36). L'acte de lire n'est d'ailleurs jamais clairement défini et, pour les responsables de l'éducation japonais, seule la maîtrise successive des différentes compétences de lecture, à partir du support unique que constitue le manuel de langue japonaise, permet d'améliorer progressivement la capacité de lire et de comprendre des enfants.

Sur ce dernier point pourtant, comme sur ceux que l'on va voir à présent, la «méthode » que nous avons décrite relève bien d'une série de choix pédagogiques effectués dans le passé, et non de ce qu'impose la langue écrite.

\subsection{Les raisons d'une «méthode »...}

Ainsi, le fait de commencer l'apprentissage de la lecture et de l'écriture par les kana est-il le fruit de décisions prises au début de l'ère Meiji (1868-1912), à partir de l'observation des méthodes d'enseignement de la lecture utilisées dans les pays occidentaux. Jusque-là, en effet, c'étaient les kanji qui étaient enseignés en premier, les kana étant au mieux enseignés en parallèle au début de l'apprentissage de 
ceux-ci5. II en va de même de l'ordre d'enseignement des kanji qui part des plus simples graphiquement et des plus fréquents dans les écrits quotidiens, pour aller vers les plus complexes et les moins fréquents. Auparavant, on ne tenait aucunement compte de ces deux critères, les caractères étant enseignés dans leur ordre d'apparition dans les textes qui étaient utilisés comme supports (en général des classiques chinois ou japonais), ou bien encore, en fonction de leur importance culturelle ou morale (kanji entrant dans la transcription des noms des empereurs ou des ères, ou encore des vertus du confucianisme, etc.). L'idée de limiter le nombre de kanji par année d'étude date aussi de la même époque. On chercherait toutefois en vain quelque étude qui justifie ou valide ces choix passés ou plus récents : les enfants ne pourraient-ils pas en étudier davantage? Plus rapidement? Mieux? Ou alors ne devraient-ils pas en étudier moins? Difficile de se faire une idée au-delà des discours programmatiques qui justifient ces choix.

On comprend bien, par ailleurs, que la progression qui part des hiragana pour aller vers les kanji a été retenue parce que la simplicité de la relation phonie-graphie qui caractérise les premiers permet de venir à bout de la difficulté inhérente aux seconds, en rendant possible l'étalement de leur apprentissage dans le temps, sans que cet étalement soit un obstacle aux activités de lecture. Toutefois, rien ne prouve qu'un apprentissage simultané ou inversé serait moins cohérent sinon moins efficace. Rares sont ceux qui, à l'instar d'un Ishii Isao (1919-2004), ont remis en cause la progression officielle fondée sur l'apprentissage préalable des kana et l'étude ultérieure et progressive des kanji. Pour le spécialiste (controversé) de l'enseignement des kanji qu'était Ishii, par exemple, les kanji sont si «faciles» et les capacités cérébrales des jeunes enfants si «prodigieuses » qu'il faudrait absolument faire débuter leur apprentissage le plus tôt possible et en faire assimiler aux enfants un maximum pendant leurs plus jeunes années. Sans souscrire à ses positions excessives et élitistes, il est clair toutefois que la critique d'Ishii faisait et continue de faire mouche sur un point au moins : le fait que la méthode actuelle ne repose sur aucune justification pédagogique, psychologique ou linguistique, mais sur l'idée qui avait cours à la fin du XIXe siècle qu'il fallait partir de ce qui était simple pour aller vers le complexe : «ce qui était simple » étant bien sûr décidé par les adultes au regard, si l'on y songe bien, des seuls critères graphiques.

Le japonais d'aujourd'hui s'écrivant en mêlant kana et kanji, on peine à trouver une raison qui s'opposerait à ce que l'enseignement de la lecture débute à partir d'écrits mêlant ces deux types de signes, dans des proportions et en suivant

5. Certes durant l'époque d'Edo (1603-1868), qui précède l'ère Meiji, l'état de la langue est différent de celui qui sera le sien à la fin de cette dernière, notamment du fait de la prédominance dans les écrits officiels du kanbun, c'est-à-dire du chinois... écrit par les Japonais. La langue utilisée hors des milieux officiels possède cependant elle, déjà, son caractère mixte alors que l'enseignement des kanji et celui des kana reste séparé, le premier étant considéré comme noble, et le second, comme vulgaire ou populaire (Galan, 1998, 1999a, 1999b). Jusqu'à l'ère Meiji, toutefois, du point de vue de l'apprentissage de l'écrit, l'enseignement des kanji restera le plus souvent premier par rapport à celui des kana. les conventions qui sont celles ayant actuellement cours dans la société. Points corollaires, rien dans la nature particulière du système d'écriture japonais n'impose a priori que tous les enfants parviennent à l'écrit par le même cheminement et au même moment, de même que rien n'impose que la seule méthode possible soit aussi systématique, mécanique, et synthétique que celle qui est effectivement en vigueur.

L'avantage des hiragana est évident, mais les problèmes qu'entraîne leur présentation au tout début de la scolarité le sont autant : pourquoi, peut se demander un enfant japonais de l'école élémentaire, s'efforcer de mémoriser des signes aussi complexes que les kanji, quand tout peut être écrit en hiragana? Les enfants chinois ne se posent jamais, eux, cette question, car sans une bonne connaissance des caractères, aucun texte ne leur est accessible. Ils ont d'ailleurs toujours, dans les comparaisons internationales, de meilleurs résultats concernant la maîtrise des caractères chinois que n'en ont les petits Japonais concernant celle des kanji.

Le fait de partir d'une langue écrite aussi «pédagogiquement simplifiée", et, au regard du moins de la langue adulte, aussi artificielle, ne pourrait-il pas être l'un des principaux facteurs à l'origine des réactions de rejet que l'on observe chez beaucoup d'enfants japonais à l'égard de la lecture et des kanji? Une réalité qui transparaît non seulement dans les diverses enquêtes menées à ce sujet, mais également dans les réactions de nombreux enseignants lorsqu'ils déclarent: «les enfants n'aiment pas les kanji, ils n'aiment pas les manuels non plus, et pourtant il faut bien faire avec! » (Galan, 2001a : 305-308).

\section{3. "Stratégie», «progression», «méthode», «pratiques pédagogiques»}

En réalité, de quoi parle-t-on au Japon, Iorsqu'on parle de «méthode» d'enseignement de la lecture?

Le lecteur l'aura sans doute pressenti dans les lignes qui précèdent : il est très délicat de cerner ce que l'on peut qualifier de «méthode", et nous sommes nous même très gêné lorsqu'il s'agit d'utiliser ce terme dans un tel contexte, car il faut alors choisir entre ce que, en tant qu'observateur étranger, on qualifierait spontanément de «méthode ", et ce qu'un Japonais désigne, lui, par ce terme - les deux ne coïncidant pas. C'est pourquoi on a eu recours un certain nombre de fois ici, de manière sans doute pas toujours convaincante, aux termes de "stratégie ", «progression ", etc. Ce que l'on qualifie en effet de « méthode » d'un point de vue français n'est pas à l'évidence, du moins en ce qui concerne l'enseignement de la lecture, ce que les Japonais eux-mêmes désignent par les termes hôhô, shidôhô ou encore oshiekata, que les dictionnaires donnent pourtant comme des traductions de «méthode» ou «méthode d'enseignement ».

Ces mots en effet ne s'appliquent au Japon, lorsque l'on considère les corpus de textes pédagogiques ou curriculaires où ils apparaissent, que pour désigner les pratiques ou les procédés pédagogiques les plus concrets que les enseignants mettent en place dans leurs classes. 
Des différentes acceptions du terme «méthode 》 (Dictionnaire encyclopédique de l'éducation, 660-666), l'usage japonais a en fait retenu celui qui est le plus étroit, le plus concret, le plus instrumental, à savoir ce qui relève de la décision ou du choix des enseignants. Par ailleurs, dans le cadre de l'enseignement de la lecture, cet usage «exclut » les définitions plus larges ou plus globales. Ainsi ce terme n'englobe-t-il jamais dans ce cadre-là la progression : «mémorisation préalable des hiragana» > «mémorisation-accumulation des kanji» > «substitution progressive des kanji aux kana», pas plus qu'on ne le trouve pour désigner le fait que la pratique des enseignants ne peut se dérouler que dans le cadre contraint des directives officielles, des manuels scolaires autorisés et des pratiques concertées et collectives des enseignants de chaque niveau d'une école donnée. Tout cela ne fait pas «méthode» au Japon. Ce qui fait «méthode », c'est ce qu'il reste à mettre en place une fois ce cadre posé. C'est-à-dire ce que désigne également un autre terme, kufû, souvent utilisé à ce niveau. On peut le traduire par « invention », mais il désigne ici tout ce qu'un enseignant va pouvoir ou devoir «inventer » comme procédé dans le cadre restreint de ces contraintes, jamais donc définies, elles, comme faisant partie de la «méthode».

Ce cadre qui résulte de choix passés apparaît comme un contexte incontournable qui n'est d'ailleurs jamais discuté. L'importance accordée à la répétition, à la récitation (au par cœur), la pratique intensive et systématique de la lecture à haute voix, ou encore le lien très fort existant entre la pratique scolaire de la lecture et l'enseignement de la morale, qui ne sont véritablement compréhensibles que replacés dans le contexte historique de l'évolution ayant conduit à la situation actuelle, ne font pas non plus «méthode ", et se posent eux aussi en «invariants » ou en «évidence » de l'enseignement de la lecture au Japon. Il en va de même de l'acceptation tacite par tous - parents, enseignants, spécialistes - de ladite méthode comme la seule possible, et de l'absence totale ou presque de débats à son sujet. Tout au plus, donc, trouve-t-on ainsi des discussions sur les pratiques concrètes à mettre en place dans les classes, qui constituent d'ailleurs l'essentiel des ouvrages pédagogiques japonais consacrés à la lecture.

D'ailleurs, si l'on examine dans le détail les directives directement liées à l'apprentissage de la lecture dans les programmes d'enseignement officiels depuis les années 1950 jusqu'à nos jours, on se rend compte que la seule variable sur laquelle le ministère de l'Éducation ait jamais véritablement joué jusqu'ici pour «améliorer » l'enseignement et la pédagogie est la variable quantitative du volume horaire attribué à la matière langue japonaise : soit qu'il augmente celui-ci, soit qu'il le diminue en fonction des débats du moment. Mais sans toucher à «tout le reste ».

La «méthode » d'enseignement, non définie en tant que telle, relève ainsi de plusieurs choix éducatifs qui n'ont jamais été vraiment explicités, ou évalués, mais qui en revanche, au moins depuis la fin des années 1950, ont toujours été présentés comme s'ils étaient les seuls possibles. Des choix, qui, historiquement - comme on l'a vu -, ont été effectués à partir de préoccupations qui étaient loin d'avoir toutes pour objet l'apprentissage de la lecture, et sans que l'on ait véritablement cherché à mettre à jour les processus d'apprentissage par lesquels les apprentis lecteurs japonais s'appropriaient la lecture, ni à en évaluer les performances. Bref, le modèle pédagogique retenu est peu en rapport avec les connaissances scientifiques du moment, ou plutôt, s'étant structuré dans le passé sur d'autres critères, il s'est développé et demeure encore aujourd'hui en dehors de celles-ci.

\section{LES EFFETS DE L'ENQUÊTE PISA SUR LA MÉTHODE D'ENSEIGNEMENT DE LA LECTURE}

II y a maintenant une dizaine d'années, nous écrivions au sujet de l'enseignement de la lecture au Japon :

«Le problème actuel de l'enseignement de la lecture au Japon est qu'on ne peut le réformer [...] sans passer par une réforme complète, non seulement de l'école élémentaire, mais aussi du système éducatif tout entier. Si les critiques qui se multiplient à l'encontre du système dans son ensemble laissent présager, à plus ou moins long terme, une crise du type de celle qu'a connue la France au début des années 1960 au sujet des missions et de l'efficacité de l'école élémentaire, il semble toutefois peu probable qu'au Japon cette crise se focalise sur l'enseignement de la lecture [...]. » (Galan, 2001a : 337)

Et de fait, cette remise en cause de l'école publique japonaise a bien eu lieu, qui a abouti à partir des années 2000 à toute une série de réformes, que nous ne pouvons pas aborder ici6, mais qui, dans un premier temps, n'ont effectivement guère touché l'enseignement de la lecture tel que nous l'avons décrit dans cet article. Si ce n'est au travers de la baisse du volume horaire accordé à la matière «langue japonaise » lors de la révision des directives officielles d'enseignement de 1998.

Ce que nous n'avions pas anticipé toutefois c'est que, à peine ces directives allaient-elles être complètement entrées en vigueur, en 2002, que l'enseignement de la lecture allait revenir, pour la première fois depuis bien longtemps, au centre des débats sur l'école. Non pas au travers d'une remise en cause des fondements de la méthode retenue. Non pas au vu de résultats d'études qui auraient montré comment les enfants japonais parvenaient réellement à la maîtrise de la lecture. Pas plus encore du fait de la prise en compte des derniers travaux de psycho ou neurolinguistique concernant l'acquisition des kana et des kanji. Non, rien de tout cela, mais... du fait des résultats des élèves japonais aux différentes enquêtes PISA.

PISA 2000, dont l'un des objectifs principaux était d'évaluer la "compréhension de l'écrit", avait inquiété en montrant que les scores des élèves japonais étaient inférieurs, entre autres, à ceux des élèves de Finlande (1re avec un score moyen de 546) et de Corée du Sud (Nanba, 2006). Le Japon se classait pourtant à la 8 e place générale pour la compréhension de l'écrit (522) avec, par rapport aux diverses compétences évaluées, la 6e place pour la capacité

6. Sur cette question voir Galan et Lévi Alvarès (2012) 


\begin{tabular}{|c|c|c|c|c|c|c|c|c|c|c|}
\hline \multicolumn{4}{|c|}{ Score/rang en lecture-compréhension } & \multirow[t]{2}{*}{ Japon } & \multicolumn{6}{|c|}{ Niveaux de compétence en lecture-compréhension } \\
\hline $\begin{array}{l}\text { Localiser et } \\
\text { extraire des } \\
\text { informations }\end{array}$ & $\begin{array}{l}\text { Intégrer et } \\
\text { interpréter }\end{array}$ & $\begin{array}{l}\text { Réfléchir } \\
\text { et évaluer }\end{array}$ & $\begin{array}{c}\text { Résultat } \\
\text { global }\end{array}$ & & $\begin{array}{c}\text { Sous le } \\
\text { niveau } 1\end{array}$ & $\begin{array}{c}\text { Niveau } \\
1\end{array}$ & $\begin{array}{c}\text { Niveau } \\
2\end{array}$ & $\begin{array}{c}\text { Niveau } \\
3\end{array}$ & $\begin{array}{l}\text { Niveau } \\
\quad 4\end{array}$ & $\begin{array}{c}\text { Niveau } \\
5\end{array}$ \\
\hline $526 / 6 e$ & $518 / 8^{e}$ & $530 / 5^{e}$ & $522 / 8 e$ & 2000 & $2,7 \%$ & $7,3 \%$ & $18,0 \%$ & $33,3 \%$ & $28,8 \%$ & $9,9 \%$ \\
\hline- & - & - & $498 / 14 \mathrm{e}$ & 2003 & $7,4 \%$ & $11,6 \%$ & $20,9 \%$ & $27,2 \%$ & $23,2 \%$ & $9,7 \%$ \\
\hline- & - & - & $498 / 15^{e}$ & 2006 & $6,7 \%$ & $11,7 \%$ & $22,0 \%$ & $28,7 \%$ & $21,5 \%$ & $9,4 \%$ \\
\hline $530 / 4 \mathrm{e}$ & $520 / 7 e$ & $521 / 8 e$ & $520 / 8 e$ & 2009 & $4,7 \%$ & $8,9 \%$ & $18,0 \%$ & $28,0 \%$ & $27,0 \%$ & $13,4 \%$ \\
\hline
\end{tabular}

(*) Le «niveau 1 » est le niveau de maîtrise le plus bas et le «niveau 5 » le plus élevé

Figure 3.

Les performances en lecture-compréhension des élèves japonais dans les enquêtes PISA 2000, 2003, $2006,2009$.

\begin{tabular}{|c|c|c|c|c|c|c|c|c|c|c|}
\hline \multicolumn{4}{|c|}{ Score/rang en lecture-compréhension } & \multirow[t]{2}{*}{ France } & \multicolumn{6}{|c|}{ Niveaux de compétence en lecture-compréhension } \\
\hline $\begin{array}{l}\text { Localiser et } \\
\text { extraire des } \\
\text { informations }\end{array}$ & $\begin{array}{l}\text { Intégrer et } \\
\text { interpréter }\end{array}$ & $\begin{array}{l}\text { Réfléchir } \\
\text { et évaluer }\end{array}$ & $\begin{array}{c}\text { Résultat } \\
\text { global }\end{array}$ & & $\begin{array}{c}\text { Sous le } \\
\text { niveau } 1\end{array}$ & $\begin{array}{c}\text { Niveau } \\
1\end{array}$ & $\begin{array}{l}\text { Niveau } \\
2\end{array}$ & $\begin{array}{c}\text { Niveau } \\
3\end{array}$ & $\begin{array}{c}\text { Niveau } \\
4\end{array}$ & $\begin{array}{c}\text { Niveau } \\
5\end{array}$ \\
\hline $515 / 10 e$ & $506 / 13 e$ & $496 / 17 e$ & $505 / 14 e$ & 2000 & $4,2 \%$ & $11,0 \%$ & $22,0 \%$ & $30,6 \%$ & $23,7 \%$ & $8,5 \%$ \\
\hline- & - & - & $496 / 17 e$ & 2003 & $6,3 \%$ & $11,2 \%$ & $22,8 \%$ & $29,7 \%$ & $22,5 \%$ & $7,4 \%$ \\
\hline- & - & - & $488 / 23 e$ & 2006 & $8,5 \%$ & $13,3 \%$ & $21,3 \%$ & $17,9 \%$ & $21,8 \%$ & $7,3 \%$ \\
\hline $492 / 26 e$ & $497 / 20$ e & $495 / 23 e$ & $496 / 22 e$ & 2009 & $7,9 \%$ & $11,8 \%$ & $21,1 \%$ & $27,2 \%$ & $22,4 \%$ & $9,6 \%$ \\
\hline
\end{tabular}

Figure 4.

Pour comparaison, voici les résulats pour la France.

à retrouver de l'information (526), la 8e place pour l'interprétation (518), et la $5^{\mathrm{e}}$ place pour la réflexion et l'évaluation (530) (voir fig. 37 ). Des résultats dont beaucoup de nations auraient pu se féliciter. Pourtant c'est une lecture «négative» qui fut faite de ces résultats par les autorités éducatives elles-mêmes, lançant dans l'archipel le débat sur la baisse des connaissances ${ }^{8}$.

Ce n'était rien toutefois à côté de ce qu'allaient déclencher PISA 2003 puis PISA 2006 avec des performances encore plus «catastrophiques» pour le Japon et, notamment, la rétrogradation du pays respectivement à la 14 e et à la 15 e place pour la compréhension de l'écrit (voir fig. 3). Dès lors le débat cessa de porter sur la nécessité ou non de réformer, pour s'intéresser au contenu des mesures à prendre en urgence, pour se concentrer sur ce qu'il fallait effectivement réformer, tout le monde étant à présent convaincu que la baisse du niveau des compétences des enfants japonais était réelle et grave. Et de fait, c'est l'allégement horaire et programmatique qui avait été au centre de la précédente révision décennale des directives officielles (1998) qui fut tenu pour principal cause du désastre.

7. À titre de comparaison, les performances en lecture-compréhension des élèves français lors de ces mêmes enquêtes sont montrées ci-dessus dans la figure 4 .

8. Certes, mais nous laisserons de côté ici cette question, PISA tombait également à pic en ces années pour permettre à ceux qui, dans l'archipel, prônaient une réforme néolibérale du système éducatif public, de noircir le plus possible la situation afin de justifier les mesures les plus radicales qu'ils entendaient prendre.
En décembre 2005, le ministère de l'Éducation lança un «Programme pour améliorer les compétences en lecturecompréhension » (Dokkairyoku kôjô puroguramu) qui imposait de faire de cette amélioration la priorité de toutes les matières (et pas seulement de la matière "langue japonaise ") et de toutes les années scolaires de l'école élémentaire au collège. Trois ans plus tard, dans la lignée de ce programme, un haut responsable du ministère de l'Éducation cité par le journal Yomiuri du 21 octobre 2008, déclarait à ce sujet : «Dans les nouvelles directives d'enseignement également [publiées cette année-là], il est demandé de faire en sorte de développer les compétences linguistiques qui concernent profondément la capacité de lire et comprendre, non seulement dans la matière langue japonaise, mais aussi dans toutes les autres matières. " Et de fait, les directives de 2008, qui sont appliquées dans toutes les années de l'école primaire depuis 2011, et le seront à partir de 2012 au collège, peuvent être considérées comme ayant été rédigées pour répondre aux besoins de PISA avec l'introduction d'objectifs centrés sur les compétences évaluées par cette enquête. On trouve ainsi, dans les remarques générales du document officiel qui accompagne et explique les nouvelles directives de l'école élémentaire pour la matière "langue japonaise" (Shôgakkô gakushû shidô yôryô kaisetsu - kokugo hen, juin 2008), les lignes suivantes qui expliquent la philosophie des nouvelles directives:

«Par ailleurs, concernant les écoliers et les collégiens de notre pays, on a pris en compte, à partir des différentes enquêtes 
telles que l'enquête PISA de l'OCDE, des choses telles que : 1) la compréhension écrite et la capacité à décrire qui réclament des capacités de réflexion, de jugement et d'expression, ainsi que l'application des connaissances et des compétences; 2) la dispersion de la répartition des scores en lecture-compréhension qui devient de plus en plus importante ainsi que les questions de motivation à apprendre, d'habitudes d'étude et d'habitudes de vie telle que le temps passé à étudier à la maison qui se trouvent à l'arrière-plan [de cette dispersion]; 3) le manque de confiance en soi, l'inquiétude dans le futur, ou encore la baisse des capacités physiques. 》

Tous ces points étant des éléments que tente d'évaluer PISA, les directives de 2008 ont bel et bien été rédigées, sans doute pour l'une des toutes premières fois au Japon, du moins aussi ouvertement, pour satisfaire à des «critères internationaux ${ }^{9}$ ».

Pourtant, lorsqu'on revient aux directives elles-mêmes, on ne trouve guère de modifications importantes en ce qui concerne l'enseignement de la lecture, sinon, comme on l'a déjà évoqué, la mobilisation de toutes les disciplines pour développer les capacités des élèves en lecturecompréhension et l'augmentation du volume horaire de la matière langue japonaise qui est passé de 1377 à 1461 périodes (+ 84 soit $+6 \%$ ) sur l'ensemble des 6 années de l'école élémentaire et de 350 à 385 (+ 35 soit $+10 \%)$ sur l'ensemble des 3 années du collège. Cela montre que c'est essentiellement, une nouvelle fois, sur la variable temps que le ministère a joué... sans toucher au fond même de la méthode que nous avons décrite dans cet article.

La publication des résultats de PISA 2009 a été marquée par une remontée spectaculaire du Japon qui a retrouvé en lecture-compréhension son rang de 2000 (8e) (voir fig. 3). $\mathrm{Ce}$ qui a, semble-t-il, mis un terme à la crise qui avait commencé avec PISA 2003.

Pourtant plusieurs questions restent en débat. Concernant les performances des élèves japonais en lecture-compréhension tout d'abord. En effet, si les performances moyennes des élèves japonais ont retrouvé leur niveau de 2000, les écarts se sont creusés entre les meilleurs résultats et les moins bons $(+3,6 \%$ pour les niveaux $<1$ et 1 ; et $+1,7 \%$ pour les niveaux $<4$ et 5 ). Comme dans d'autres pays, il y aurait donc au Japon aussi, aujourd'hui, une augmentation du nombre des bons lecteurs mais également de plus en plus d'enfants ayant de grandes difficultés à lire. Constat «banal » dans d'autres pays, c'est là une situation vraiment nouvelle dans l'archipel, du moins en ce qu'elle amène les Japonais à prendre conscience que, contrairement à l'idée qui dominait jusque-là, tous les enfants ne parviennent pas à un niveau de maîtrise de la lecture sinon identique du moins équivalent. Les résultats de PISA 2009 ayant été publiés fin 2010-début 2011, il est toutefois encore trop tôt pour voir quelles conséquences concrètes ils auront au Japon en termes de politique éducative.

9. Le Japon n'avait guère réagi auparavant aux enquêtes nationales ou internationales qui allaient dans le même sens en ce qui concerne la lecture-compréhension ; voir notamment l'accueil réservé aux enquêtes comparatives de Harold W. Stevenson dans les années 1980-1990 (Galan, 2001a : 307-308)

\section{CONCLUSION}

Que dire pour conclure? II faut tout d'abord insister sur le fait que la méthode d'enseignement de la lecture appliquée aujourd'hui dans les écoles japonaises, et que nous avons décrite dans cet article, s'inscrit dans la droite ligne de celle progressivement mise en place, sur le modèle des méthodes syllabiques, word method et autre sentence method occidentales, à la fin du XIXe siècle, et qui se posait, elle, en rupture par rapport aux pratiques qui avaient cours auparavant. Elle en partage les mêmes caractéristiques fondamentales et on chercherait en vain au cours du siècle passé une quelconque nouvelle rupture qui aurait fait évoluer cet enseignement dans une autre direction. Durant toutes ces années, et notamment au cours du demi-siècle passé, l'enseignement de la lecture n'aura ainsi connu au Japon, à la différence de la plupart des pays occidentaux, aucun débat sur le code et le sens, aucune crise, aucune querelle de méthodes, aucun débat sur la montée de l'illettrisme (qui n'y est de toute façon pas mesuré), aucune influence des sciences cognitives, etc. Si les conflits ou débats théoriques entre spécialistes existent pourtant bel et bien au Japon, ils portent sur l'école et les pratiques pédagogiques en général et ne se focalisent en rien sur ce seul enseignement. L'enseignement de la lecture s'est, de fait, dans ce pays, complètement structuré au cours du siècle écoulé en dehors de la recherche. Et il reste toujours aujourd'hui prisonnier d'une telle situation : les chercheurs qui s'intéressent à cet enseignement avouent la plupart du temps n'avoir aucune influence sur les directives du ministère, ni sur le contenu des manuels, abandonnés aux fonctionnaires du ministère de l'Éducation, et, de fait également, très peu d'influence sur les pratiques des enseignants.

Cet immobilisme et le caractère d'évidence dont est parée la méthode d'enseignement de la lecture appliquée depuis des décennies ont résisté à toutes les alertes qu'il a pu y avoir et qui ont pu montrer que les enfants japonais n'avaient peut-être pas une aussi excellente maîtrise de la lecture que ne le prétendait le ministère et ne le croyait l'opinion publique. Cette situation n'a changé qu'avec PISA 2000, et surtout 2003, dont les résultats ont, comme on l'a vu, pour la première fois depuis des décennies, obligé les responsables et les spécialistes à reconsidérer la méthode en vigueur. $\mathrm{Ou}$, plus exactement, non pas la méthode en vigueur, mais les évolutions curriculaires et horaires qui avaient affecté l'enseignement de la matière "langue japonaise " les années précédant la réalisation des enquêtes PISA. Et sans que l'on puisse dire, à ce jour, si ce mouvement de réflexion vers le changement va se poursuivre ou non, et s'il conduira à une remise en cause plus profonde des choix effectués en la matière il y a plus d'un siècle. La remontée des scores des élèves japonais lors de PISA 2009 ayant rassuré responsables et opinion publique, la poursuite ou non de la réflexion sur le sujet pourrait bien dépendre de ce que dira PISA 2012...

Enfin, et ce sera notre conclusion, cette remontée significative du Japon ne laisse toutefois pas d'interroger sur ce que PISA mesure véritablement. Ou, si on le dit autrement, 
sur la manière dont le Japon a pu en six années inverser la tendance et revenir à son point de départ en matière de performances en lecture-compréhension. Certes, on ne peut que se réjouir de voir que les tendances négatives que met à jour PISA peuvent être inversées en un aussi court laps de temps. Et beaucoup de pays pourraient donc s'inspirer de ce qui a été fait au Japon. Toutefois, et c'est là justement que l'on peut être perplexe : qu'a fait exactement le Japon durant ces six années pour parvenir à redresser ses résultats? Nous nous garderons bien de répondre ici, car cela mérite à l'évidence d'être étudié de beaucoup plus près que nous n'avons pu le faire. Une telle interrogation nous semble toutefois légitime, étant donné que la plupart des mesures que nous avons évoquées dans notre dernière section n'ont au mieux été appliquées que pendant 3 ans, en ce qui concerne les élèves de 15 ans concernés par PISA 2009, ce qui est très court en termes d'efficacité d'une politique éducative. Quant à la révision des directives de 2008, elle n'avait même pas commencé à être mise en place au moment où les épreuves de PISA 2009 ont été passées.

Quels ont donc été les facteurs qui ont permis ce spectaculaire rétablissement? La question, pour nous, reste ouverte. Avec comme corollaire celle qui consiste à se demander si l'on peut ainsi améliorer aussi rapidement les performances réelles des élèves en matière de lecture-compréhension (et si oui comment) ou s'il est juste possible de mieux préparer les élèves à réussir le type d'épreuves sur lesquelles repose PISA.

\section{Christian GALAN Université de Toulouse-le Mirail CEJ-Inalco}

\section{RÉFÉRENCES BIBLIOGRAPHIQUES}

Frellesvig B. (2010), A History of the Japanese Language, Cambridge, University of Cambridge.

Blin R., TAmba I. (éds.), Faits de langue, $\mathrm{n}^{\circ} 17$ «Coréenjaponais ".

GALAN C. (1995a), «L'Importance de l'«environnement » dans l'apprentissage de la lecture au Japon ", Psychologie et Éducation, $n^{\circ} 20$, p. 49-69.

GALAN C. (1995b), «Japon : l'enseignement de la lecture dans le cas d'une écriture mixte - problématique et politique ", Revue française de pédagogie, $n^{\circ} 113$, p. 5-18.
GALAN C. (1998), «L'enseignement de la lecture à la veille de la promulgation du Gakusei (1872) - La méthode classique », Ebisu - Études japonaises, $n^{\circ} 18$, p. 5-47.

GALAN C., (1999a), "Les manuels de langue au lendemain de la Restauration de Meiji - Les innovations de la période du décret sur l'éducation ", Cipango - Cahiers d'études japonaises, $\mathrm{n}^{\circ} 8$, p. 215-257.

GALAN C. (1999b), "L'ébauche d'un nouvel enseignement de la langue écrite à la veille des réformes éducatives de 1872 ", Ebisu - Études japonaises, $n^{\circ} 22$, p. 77-124.

GALAN C. (2001a), L'Enseignement de la lecture au Japon - Politique et éducation, Toulouse, PUM, $365 \mathrm{p}$.

GaLAN C. (2001b), «Lire le Japonais », Faits de langue, $\mathrm{n}^{\circ} 17$, «Coréen-japonais », p. 43-48.

GaLAN C. (2001c), «Kanji et kana: ce que nous apprennent les travaux de psycho et neurolinguistique ", Japon pluriel 4, Arles, Philippe Picquier, p. 109-122.

GALAN C. (2003), Introduction à l'écriture japonaise, CD-Rom didacticiel (présentation de l'écrit japonais et exercices d'apprentissage des kana), Toulouse, Université du Mirail.

GaLAN C. (2006), «Le(s) discours sur l'illettrisme dans le Japon contemporain ", in C. Galan et J. Fijalkow (éds.), Langue, lecture et école au Japon, Arles, Philippe Picquier, p. 341-392.

GALAN C. (2008), "L'«orthographe » mixte du japonais : le libre choix du scripteur », in C. Brissaud, J.-P. Jaffré, J.-C. Pellat (éds.), Nouvelles Recherches en orthographe, Limoges, Éditions Lambert-Lucas, p. 53-70.

Galan C., FiJalkow J. (éds) (2006), Langue, lecture et école au Japon, Arles, Philippe Picquier.

Galan C., LÉVI Alvarès C. (éds) (2012), Les Dossiers des Sciences de l'Éducation, n 37, «Séisme éducatif au Japon », mars 2012.

KAWAKAMI S. (2006), "Le début de l'apprentissage des kanji à l'école élémentaire ", in C. Galan et J. Fijalkow (éds.), Langue, lecture et école au Japon, Arles, Philippe Picquier, p. 241-254.

NANBA H. (2006), «Quelle est cette compétence à la lecture vers laquelle il faut tendre? Réflexion basée sur les enquêtes internationales et japonaises ", in C. Galan et J. Fijalkow (éds.), Langue, lecture et école au Japon, Arles, Philippe Picquier, p. $153-169$.

SHUTÔ H. (2006), «Panorama historique des méthodes d'enseignement de la lecture utilisées dans les écoles japonaises ", in C. Galan et J. Fijalkow (éds.), Langue, lecture et école au Japon, Arles, Philippe Picquier, p. 197-220.

TAMBA I. (2006), «À la recherche des systèmes d'écriture et de lecture japonais », in C. Galan et J. Fijalkow (éds.), Langue, lecture et école au Japon, Arles, Philippe Picquier, p. 55-71.

TsuKADA Y. (2006), «Les manuels scolaires et les méthodes d'apprentissage de la lecture dans les écoles élémentaires japonaises ", in C. Galan et J. Fijalkow (éds.), Langue, lecture et école au Japon, Arles, Philippe Picquier, p. 271-285. 